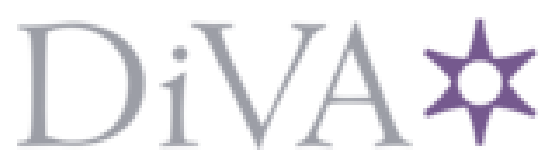

http://www.diva-portal.org

This is the published version of a paper presented at 2014 IEEE International Conference on Electronics, Computing and Communication Technologies, IEEE CONECCT 2014; Bangalore; India; 6 January 2014 through 7 January 2014.

Citation for the original published paper:

Yajnanarayana, V., Dwivedi, S., Händel, P. (2014)

Design of impulse radio UWB transmitter with improved range performance using PPM signals.

In: 2014 IEEE International Conference on Electronics, Computing and Communication

Technologies (IEEE CONECCT) (pp. 6740281-). IEEE Computer Society

http://dx.doi.org/10.1109/CONECCT.2014.6740281

N.B. When citing this work, cite the original published paper.

Permanent link to this version:

http://urn.kb.se/resolve?urn=urn:nbn:se:kth:diva-141049 


\title{
Design of impulse radio UWB transmitter with improved range performance using PPM signals
}

\author{
Vijaya Yajnanarayana, Satyam Dwivedi, Peter Händel \\ ACCESS Linnaeus Centre, \\ Signal Processing Lab, \\ KTH Royal Institute of Technology, Stockholm, Sweden \\ email:\{vpy,dwivedi,ph\}@kth.se
}

\begin{abstract}
There are several practical challenges in designing an ultra wideband (UWB) device for communication. From the physical layer signaling perspective it is important to avoid the strong peaks in the transmitted signal to fully exploit the regulatory bodies power constraint requirements. This will result in increased range performance for the sensors. Design of the transmit impulse radio (IR) UWB signals under the practical constraints of hardware and regulatory body is a critical optimization issue in the UWB system design. In this paper, we propose a IR-UWB signaling, which is a variant of pulse position modulation (PPM) method and achieves an increased range performance under the practical constraints of hardware and Federal Communications Commission (FCC) regulations.

Index terms: Ultra wideband, UWB communication, pulse position modulation, time to digital converter (TDC), peak to average power (PAPR).
\end{abstract}

\section{INTRODUCTION}

Ultra wideband (UWB) is an interesting candidate for future indoor wireless communication. In these systems a low power message signal is spread in to a wideband. Federal Communications Commission (FCC) has adopted license free UWB operations in USA [1]. This has resulted in $7.5 \mathrm{GHz}$ of spectrum available for UWB systems. Availability of large bandwidth means can enable UWB devices to communicate at very high data rates. Wide bandwidth also enables innovative system design. There is wide scope of data-rate, range and other parameters that can be traded off based on the application. Unlike its narrowband counterpart UWB systems are not power hungry and its performance in the indoor channel having dense multi paths is better. These are beneficial from the communication system design [2], [3], [4].

The use of impulse signaling for UWB was proposed by Win and Scholtz in 1990s. Their work published in [5], [6], [7] contributed significantly towards adaptation of this technology for UWB. Impulse radio signal can be seen as carrier less baseband transmission, this saves cost in the transceiver hardware as it does not need superheterodyne structure. Power, range and bitrate of the transceiver can be easily traded by controlling the amplitude, duty cycle and number of pulses per bit of transmission.

Wide bandwidth used by the UWB communication systems overlap with other radio technologies. Thus the regulatory

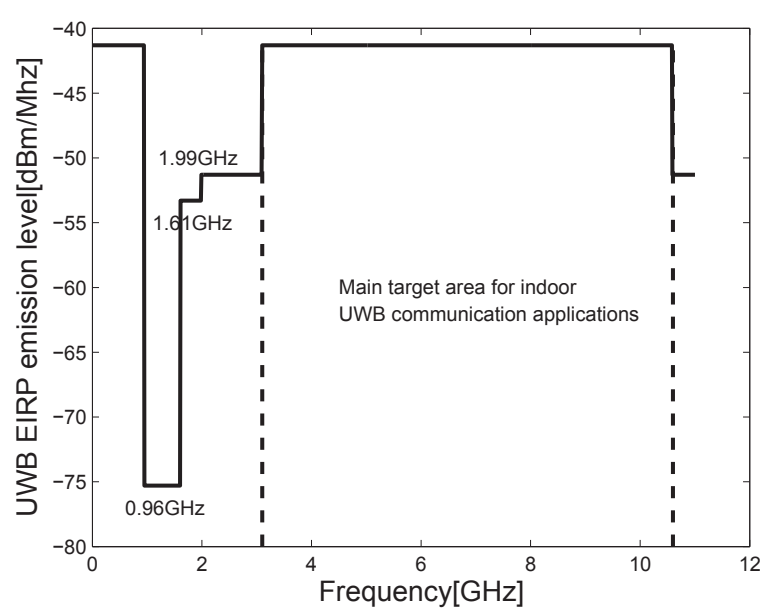

Fig. 1. Spectral mask specified by FCC for indoor UWB system.

bodies like FCC have imposed very stringent constraints on the UWB emissions in order to protect the victim receivers. These are specified through spectral masks as shown in Figure 1. High bandwidth enable the UWB transceiver to generate narrow impulse signal which can yield centimeter level accuracy for ranging. These transmitters typically generate narrow impulse signals and measure range information through round trip time (RTT). Many applications like sensor networks, asset management, healthcare, first-responder etc, require both localization and communication capabilities. Our intention is to develop UWB communication using pulse based technique so that the nodes can have both localization and communication capabilities. Constraints from low cost localization/communication hardware platform together with restrictions from regulatory body makes the UWB transmitter design challenging.

Through this correspondence, we propose a method for communication on a low cost and low power hardware architecture. We design an optimal physical layer signaling given the constraints from hardware and regulatory bodies and demonstrate the improved performance in terms of range compared to previously proposed techniques. 
This paper is organised as follows, In Section II, we discuss the pulse constrution. In Section III, we discuss the UWB transmitter hardware and design challenges. Section IV, we will discuss the modulator. Here we propose a new modulator algorithm and quatify the improvement in range compared to the previous work. Finally, in section V, we will discuss the conclusions.

\section{Pulse CONSTRUCtion FOR UWB TRANSmitters}

Transmit pulse shape plays a critical role in the emission pattern of the UWB transmitter. There are many UWB shapes explored in literature, however the one based on Gaussian pulses makes a good choice for building a cost effective UWB transceiver. The reasoning for this is that a simple transistor switching "on" and "off" to generate a narrow pulse will form this shape instead of a rectangular shape due to imperfections in micro-electronic design [8].

In [9] authors use second derivative of the scaled Gaussian pulse with zero mean and variance $\frac{\tau^{2}}{4 \pi}$ for UWB system design. This is shown in equation (2).

$$
\begin{gathered}
s(t)=-e^{-2 \pi\left(\frac{t}{\tau}\right)^{2}} \\
s^{\prime \prime}(t)=\frac{d^{2} s(t)}{d t^{2}}=-4 \pi e^{\frac{-2 \pi t^{2}}{\tau^{2}}}\left(\frac{-\tau^{2}+4 \pi t^{2}}{\tau^{4}}\right)
\end{gathered}
$$

For analytical and simulation analysis we have used the power normalized version of equation (2). This is given in equation (3).

$$
p^{2}(t)=\frac{\left(s^{\prime \prime}(t)\right)^{2}}{\int_{-\infty}^{\infty}\left(s^{\prime \prime}(t)\right)^{2}}
$$

Several modulation techniques are proposed in literature using narrow pulses [10], [7]. Primarily they are variants of PPM, binary phase shift keying (BPSK) or on-off keying (OOK). In this paper we use a variant of PPM method.

The UWB transmitter generates the PPM variant signal which is a modified pulse specified in [7] with 1 symbol per pulse and no time hopping, The transmitted output signal is given by equation (4)

$$
W_{\mathrm{tr}}(t)=\sqrt{E_{p}} \sum_{i} p\left(t-i T_{\mathrm{S}}-d_{i} \Delta-\gamma\right)
$$

where $T_{\mathrm{S}}$ is the symbol period, $d_{i}$ is the $i$ th symbol value $d_{i} \in$ $[1, . ., M], p(t)$ is the normalized pulse such that $\int_{-\infty}^{\infty} p^{2}(t)=$ $1, \sqrt{E_{p}}$ is the energy of the pulse, $\gamma$ is a parameter coming from the constraints of the typical UWB hardware which will be explained later, $\Delta$ is the modulation index and $\log _{2} M$ is the modulation order. later in the modulator design section we will see how to optimize the modulation parameter $M$ given constraints of the hardware and regulatory bodies to maximize the bitrate.

In the next section we will discuss UWB Hardware for generation and detection of these UWB pulses.

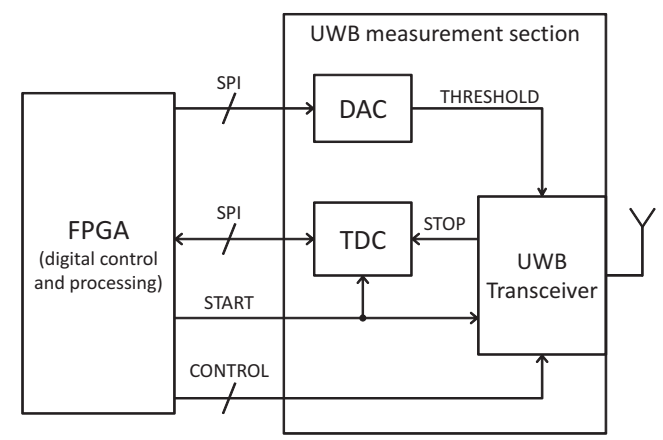

Fig. 2. UWB Sensor architecture [12]

\section{UWB HARDWARE}

The design of physical layer signal should have smooth PSD to enable transmitter to radiate large amplitude pulses there by increasing the range and detection probability with out violating spectral mask requirements. The best operating region for UWB indoor communciation nodes are $3.1 \mathrm{GHz}$ to $10 \mathrm{GHz}$ as shown in the Figure 1. In this region the average power spectral density (PSD) should not cross $-41.3 \mathrm{dBm} / \mathrm{MHz}$.

There are several techniques to generate UWB pulses at the transmitter as shown in [11], [12]. Most of the cost effective systems employ a transistor or a diode which are turned "on" and "off" to generate impulse signal.

In [12] authors propose a cost effective UWB sensor architecture platform. The sensor architecture is shown in Figure 2. This hardware consists of analog UWB measurement section, designed in-house. It has a digital processing section based on FPGA. On the transmit side, control signal triggers the diode in the transceiver "on" and "off". On the receive side the transceiver has an energy detector, which sends "Start/Stop" signal to time to digital converter (TDC). TDC measures the interval between the pulses. This ability to detect the intra-pulse duration enable the system for ranging as well demodulating PPM signals.

In general at the transmitter, it is not possible to transmit arbitrary close pulses because of the recovery time required for the micro electronic devices used in them. This creates a constraint on the signaling that the pulses need to be separated by at least by minimum distance equal to the recovery time. This is the reason for having the $\gamma$ in the equation (4).

In the subsequent sections we will show how to optimally design the modulator in view of constraints from hardware and regulatory bodies and compare its performance with the technique proposed in [9].

\section{Modulator Design}

Elimination of the strong peaks in the transmitted signal is an important aspect of the modulator design. If we use train of impulse pulses for UWB communication, The PSD of pulses will have significant peaks due to the comb formations. One 


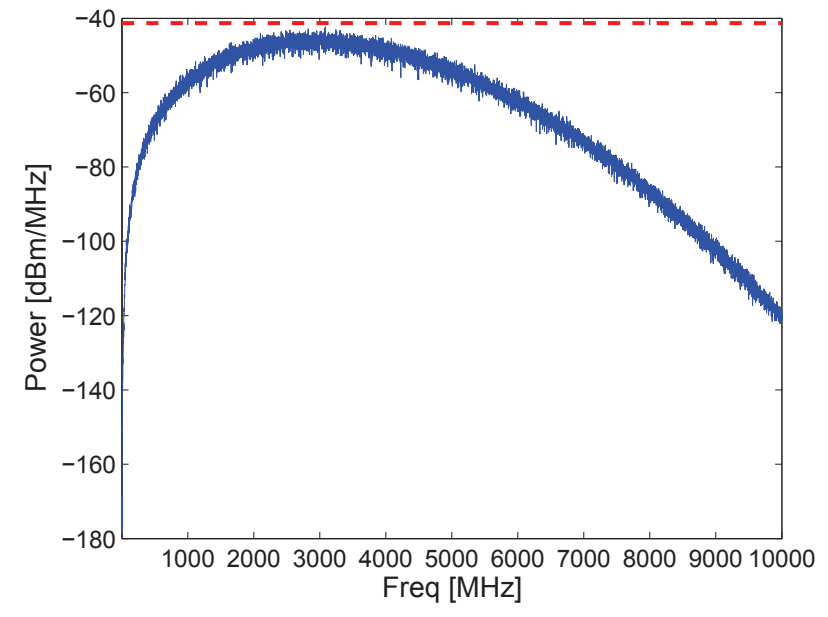

Fig. 3. PSD for IR pulses with randomized $T_{\mathrm{PRT}}\left[T_{\mathrm{S}}=10 \mathrm{~ns}\right.$ and $T=1$ ns].

way to overcome this problem is to completely randomize pulse intervals. The probability mass function (PMF) of pulse repetition frequency $T_{\mathrm{PRT}}$ is given by equation (5).

$$
\operatorname{PMF}\left(T_{\mathrm{PRT}}\right)= \begin{cases}\frac{1}{n} & \text { If } T \leq T_{\mathrm{PRT}} \leq 2 T_{\mathrm{S}} \\ 0 & \text { Otherwise. }\end{cases}
$$

Here $T$ is the pulse width of the Gaussian pulse and $n$ is the number of bins / discretized-intervals. $T_{\mathrm{PRT}}$ is varied from $T$ instead of 0 to avoid collision between pulses. With this modification average pulse duration is $T_{\mathrm{S}}$ and the comb formations are eliminated. Spectrum is smooth with out any strong peaks as shown in Figure 3. however this is not practical from the hardware perspective due to the two critical constraints from the hardware listed below.

1) At the transmit side, It is not possible to generate arbitrarily close pulses, due to the recovery time of diode. Pulses should be separated at least by $T_{\mathrm{ms}}$.

2) At the receive side, It is not possible to resolve arbitrarily close pulses. In [12] this is due to the fixed resolution $(\Delta)$ of TDC.

One way to design the signaling with these hardware constraints is to have $\gamma$ in the equation (4) equal to the minimum separation needed between the pulses (recovery time of the diode, $T_{\mathrm{ms}}$ ) and modulation index $\Delta$ in the equation (4) equal to the TDC's time resolution. Thus the resulting transmitted signal is given by equation (6)

$$
W_{\mathrm{tr}}(t)=\sqrt{E_{p}} \sum_{i} p\left(t-i T_{\mathrm{S}}-d_{i} \Delta-T_{\mathrm{ms}}\right)
$$

If $\log _{2} M$ is the modulation order then the symbol time $\left(T_{\mathrm{S}}\right)$ and bitrate $\left(R_{b}\right)$ is given by (7) and (8).

$$
\begin{aligned}
T_{\mathrm{S}} & =T_{\mathrm{ms}}+\Delta M \\
R_{b} & =\frac{\log _{2} M}{T_{\mathrm{ms}}+\Delta M}
\end{aligned}
$$

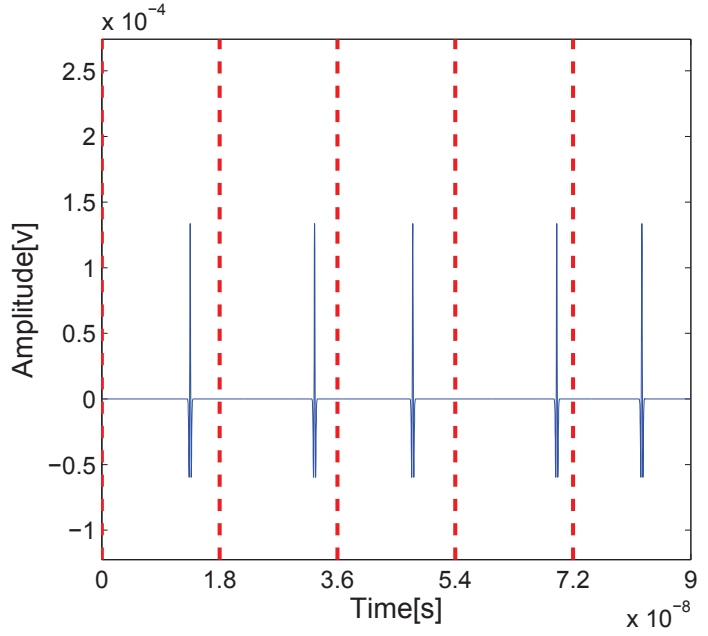

Fig. 4. PPM varient signalling in [9] to achieve maximum bit rate under the UWB hardware constraints. (Dashed lines indicate the symbol boundary)

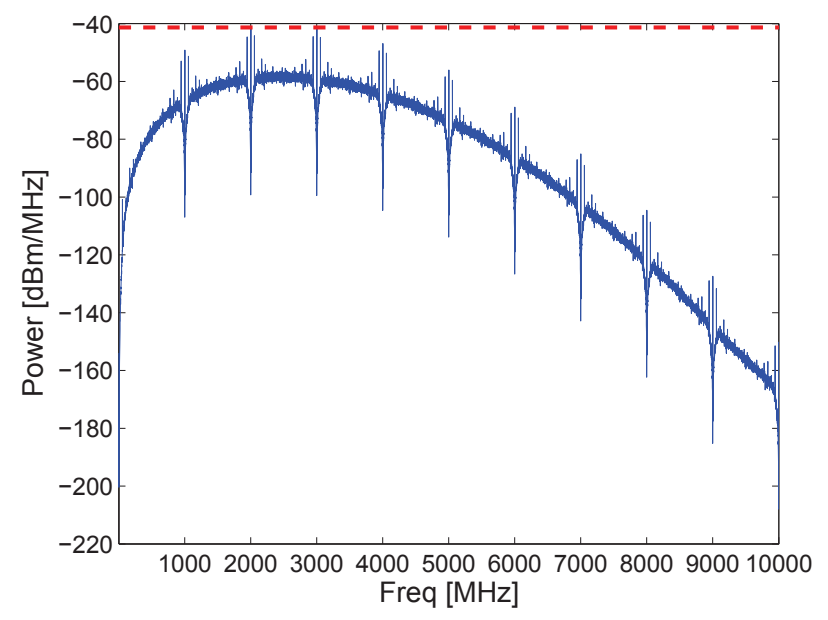

Fig. 5. PSD of signaling employed in [9].

In [9] authors propose a PPM variant signaling where modulation order is decided based on maximizing the bitrate under two hardware constraints discussed above. For the nominal values of $\left(T_{\mathrm{ms}}=10 \mathrm{~ns}\right)$ and $(\Delta=1 \mathrm{~ns}), M=8$ maximizes the bitrate. 5 pulses of this is shown in the Figure 4. Notice that each symbol duration $T_{\mathrm{S}}=18 \mathrm{~ns}$ wide. It consists of fixed gap of $10 \mathrm{~ns}$ and 3 bits of information is modulated in the remaining 8 ns. The PSD of this signaling is shown in 5 , due to the deterministic gaps in the signaling the PSD is not completely smooth.

We propose a modification to this signaling to further smoothen the PSD. Signaling employed in [9] keeps the dead time $\left(T_{\mathrm{ms}}\right)$ constant. This ensure that the minimum separation between pulses to be equal to or greater than recovery time of diode. If modulator structure is designed such that it remembers the time when it fired the pulse for previous 


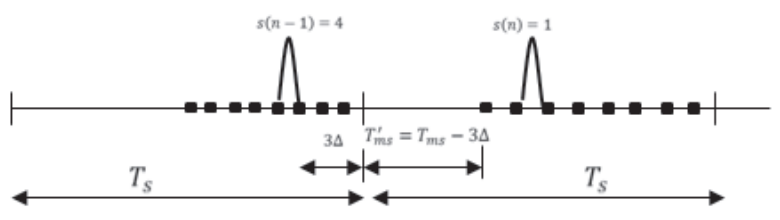

Fig. 6. Modified Signaling for UWB Modulator.

symbol, then the minimum separation needed by the next symbol can be reduced. This is illustrated in the Figure 6 . Notice that with the symbol duration $\left(T_{\mathrm{S}}\right)$ is kept constant, "dead time" $T_{\mathrm{ms}}^{\prime}$ in the subsequent symbol is reduced. In the Figure $6, T_{\mathrm{ms}}^{\prime}$ is reduced by $3 \Delta$ in the 2 nd symbol, by remembering the position of the pulse in the previous symbol. Since the duration of the symbol is constant the net result is increase in the time interval bin widths (modulation index) there by increasing the detectability. Since in this method the transmitter need to remember the past transmitted symbol to decide the $T_{\mathrm{ms}}^{\prime}$ for the current symbol, we call this signaling as with-memory signaling. The algorithm for implementing this modulator is as shown in Algorithm 1.

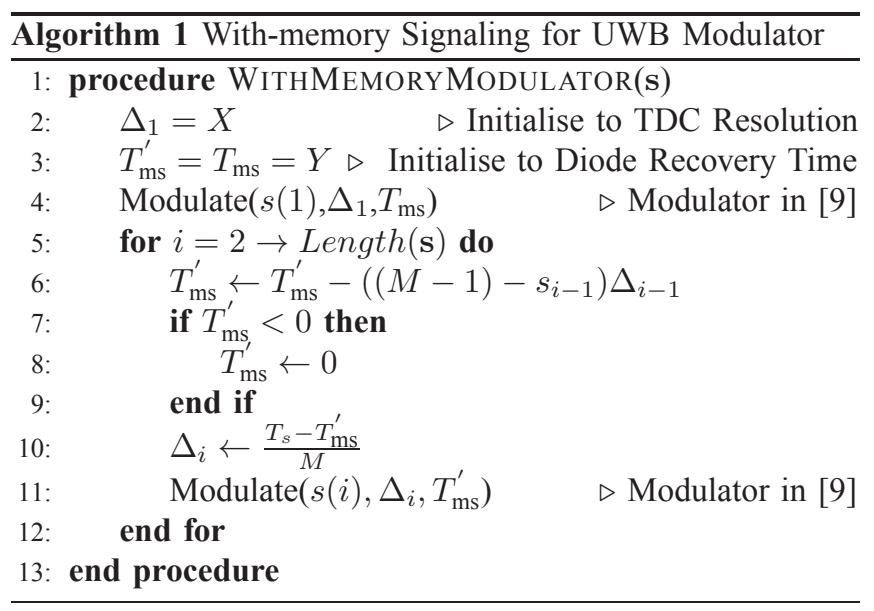

Notice in the algorithm description that the no-memory modulator of [9] is called with different bin widths $\left(\Delta_{i}\right)$ and dead time $\left(T_{\mathrm{ms}}^{\prime}\right)$. The two primary benefits from this signaling is summarized below.

1) Better detectability, This is because on an average there is an increase in the bin widths (modulation index) of possible pulse positions.

2) Better randomization of the pulses. Unlike in signaling of [9] there are no deterministic gap and pulses are spread to all regions of the symbol interval.

The impact of 2) is the further smoothing of the PSD, To assess the performance we implemented the Algorithm 1. Figure 7 shows 5 symbols in time domain and Figure 8 shows the PSD with the modified signaling of randomly generated 1000 bits. The order $M$ is chosen as 8 , recovery time $T_{\mathrm{ms}}=10 \mathrm{~ns}$ and inital modulation index $\Delta=1 \mathrm{~ns}$.

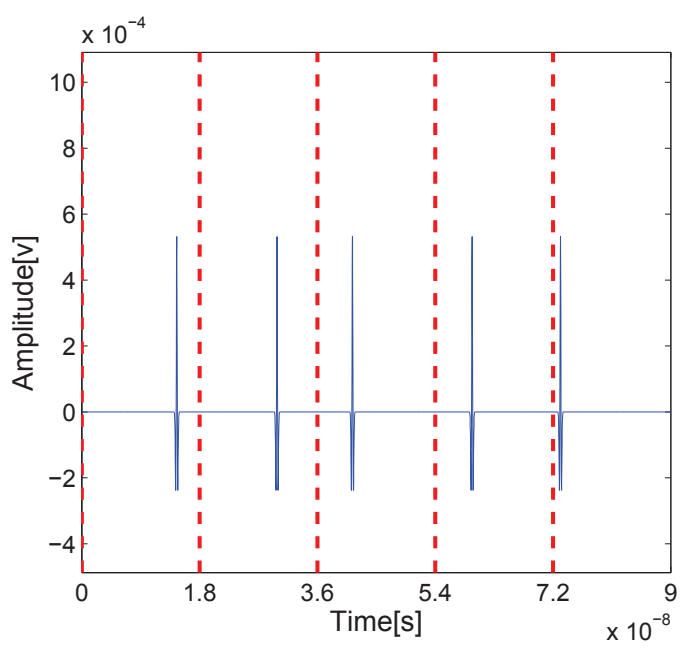

Fig. 7. Time domain waveform of the modified signaling. (Dashed line indicate the symbol boundary)

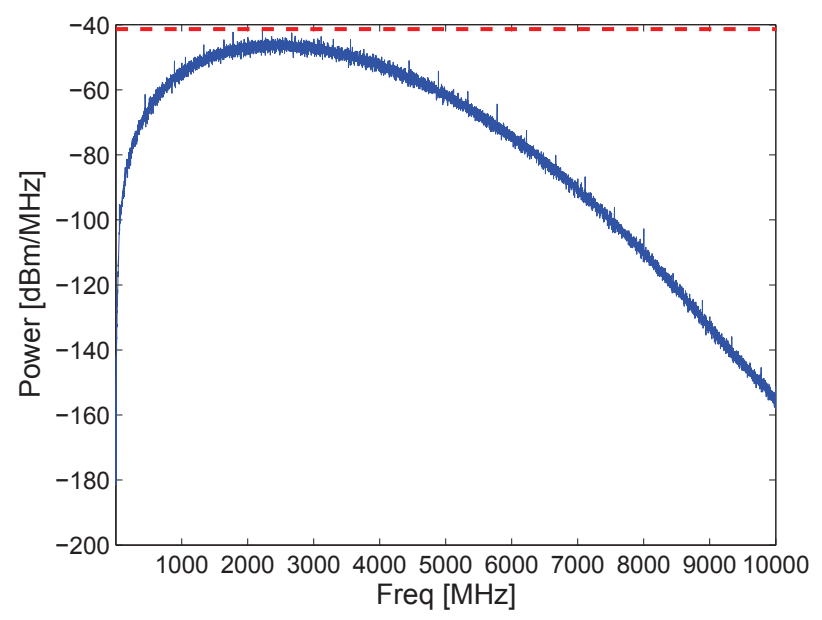

Fig. 8. PSD of the modified signaling.

Notice compared to Figure 5, There is further smoothening of the PSD which will enable the transmitter to generate pulses at higher amplitude with out violating the mask specification of the regulatory bodies there by increasing the range. Comparing the Figure 4 and Figure 7 we notice that we can increase the pulse amplitude by 4 times compared to the signaling of [9] with out violating the regulatory body specifications and with the same hardware constraints. For the line of sight (LOS) propagation model the path lass is given by the equation (9)

$$
P L=\frac{(4 \pi d f)^{2}}{c}
$$

where $f$ is the frequency of operation, $d$ is the range, and $c$ is speed of light in vacuum. For a LOS channel the received power is given by equation (10)

$$
P_{\mathrm{rx}}=\frac{P_{\mathrm{tx}}}{P L}
$$


Four times increase in amplitude results in 16 times $(12 \mathrm{~dB})$ increase in power. Since path loss is proportional to the square of the distance as given by equation (9), For any given received power and frequency of operation, if $d_{1}$ and $d_{2}$ are ranges for transmitter employing no-memory signaling of [9] and with memory signaling proposed in this paper, we have

$$
\begin{aligned}
\frac{P_{\mathrm{tx}}}{d_{1}^{2}} & =\frac{16 P_{\mathrm{tx}}}{d_{2}^{2}} \\
d_{2} & =4 . d_{1}
\end{aligned}
$$

This means that the range can be increased 4 times compared to signaling of [9]. This increased range comes with a cost of increased complexity in the modulator as shown in Algorithm 1.

\section{CONCLUSION}

In this paper, we proposed a new signaling method for UWB which avoids strong peaks in the PSD there by utilising the spectrum optimally under the constraints of the hardware. This improved spectral utilization can increase the range of the UWB nodes by 4 times compared to to the proposed method in [9]. This improved performance comes with a cost of having memory in the transmitter to remember the previous pulse position and require more computations in the modulator as proposed in the Algorithm 1.

\section{ACKNOWLEDGEMENTS}

Parts of the work have been funded by The Swedish Agency for Innovation Systems (VINNOVA).

\section{REFERENCES}

[1] "First report and order, revision of part 15 of the commision's rules regarding ultra-wideband transmission systems," tech. rep., FCC, Washington DC, ET Docket 98-153, 2002.

[2] Luecken, Communication and Localization in UWB Sensor Networks. $\mathrm{PhD}$ thesis, ETH ZURICH, 2012.

[3] J. Zhang, P. Orlik, Z. Sahinoglu, A. Molisch, and P. Kinney, "Uwb systems for wireless sensor networks," Proceedings of the IEEE, vol. 97, no. 2, pp. 313-331, 2009.

[4] A. Molisch, "Ultrawideband propagation channels-theory, measurement, and modeling," Vehicular Technology, IEEE Transactions on, vol. 54, no. 5, pp. 1528-1545, 2005.

[5] R. Scholtz, "Multiple access with time-hopping impulse modulation," in Military Communications Conference, 1993. MILCOM '93. Conference record. Communications on the Move., IEEE, vol. 2, pp. 447-450 vol.2, 1993.

[6] M. Win and R. Scholtz, "Impulse radio: how it works," Communications Letters, IEEE, vol. 2, pp. $36-38$, feb. 1998

[7] M. Win and R. Scholtz, "Ultra-wide bandwidth time-hopping spreadspectrum impulse radio for wireless multiple-access communications," Communications, IEEE Transactions on, vol. 48, no. 4, pp. 679-689, 2000.

[8] R. K. M. Ghavami, L.B. Michael, Ultra Wideband Signals and Systems in Communication Engineering. John Wiley, 2007.

[9] V. Yajnanarayana, S. Dwivedi, A. De Angelis, and P. Handel, "Design of impulse radio uwb transmitter for short range communications using ppm signals," in Electronics, Computing and Communication Technologies (CONECCT), 2013 IEEE International Conference on, pp. 1-4, 2013.

[10] K. Witrisal, G. Leus, G. Janssen, M. Pausini, F. Troesch, T. Zasowski, and J. Romme, "Noncoherent ultra-wideband systems," Signal Processing Magazine, IEEE, vol. 26, pp. 48 -66, july 2009.

[11] A. Zhu, F. Sheng, and A. Zhang, "An implementation of step recovery diode-based uwb pulse generator," in Ultra-Wideband (ICUWB), 2010 IEEE International Conference on, vol. 2, pp. 1-4, 2010.
[12] A. De Angelis, S. Dwivedi, and P. Handel, "Characterization of a flexible uwb sensor for indoor localization," Instrumentation and Measurement, IEEE Transactions on, vol. 62, no. 5, pp. 905-913, 2013. 